IASSNS-HEP-95/17

\title{
Self-Organized Critical Models Without Local Particle Conservation Laws On Superlattices
}

\author{
H. F. Chau \\ School of Natural Sciences, Institute for Advanced Study, Olden Lane, Princeton, NJ 08540, U.S.A.
}

(March 7, 1995)

\begin{abstract}
We consider simple examples of self-organized critical systems on one-dimensional superlattices without local particle conservation laws. The set of all recurrence states are also found in these examples using a method similar to the burning algorithm.
\end{abstract}

PACS numbers: 05.40.+j, 05.60.+w, 64.60.Ht 


\section{INTRODUCTION}

In 1987, Bak et al. proposed an interesting idea called self-organized criticality, suggesting that many physical systems could evolve under their own dynamics without any fine tuning parameters to states without any characteristic time and length scales [1]. They illustrated the idea using a simple cellular automaton model. Later on, their automaton model is proved to be commutative in the sense that the order of "particle addition operations" does not affect the outcome of the final state [2]. Now, this model is widely known as the Abelian Sandpile Model (ASM). In addition to the cellular automaton models, various kinds of continuous and lattice-continuous models have shown to exhibit self-organized criticality.

In particular, the self-organized critical model of dissipative transport using stochastic partial differential equation [3] has received much attention recently. This model proves that the existence of a local (particle) conservation law is not a necessary condition for the exhibition of self-organized criticality. Further discussions on the importance of particle conservation law can be found elsewhere [4]. Furthermore, the existence of local particle conservation law is not an essential feature for cellular automaton models (i.e. models on finite grid points and each grid point allows only a finite number of states) as well. The well-known forest fire model is an example of this kind [5].

In this paper, we study two new examples of Abelian Sandpile Models on one-dimensional superlattices — the simplest possible kind of self-organized critical model without local particle conservation laws. The examples we provide here can be generalized easily to higher dimensions although exact calculation of the scaling exponents and the recurrence phase space configurations is much more difficult. Studies of similar one-dimensional models can be found elsewhere [6]. A useful method, inspired by the idea of the burning algorithm [7], to find the recurrence phase space which constitute an important step in showing the criticality of our model is also introduced. This method works well for systems of low spatial dimensions.

\section{EXAMPLE 1}

Consider a collection of $2 N$ sites labeled from 1 to $2 N$ and we associate an integer $h_{i}$ called the local height to each site. Using the same rules for the Abelian Sandpile, sites with local height greater than 0 is said to be unstable and particle redistribution occurs as follows: (a) for any oddnumbered site $2 i+1$, two particles will be lost in the next timestep. Each of its two nearest neighbors, i.e. sites $2 i$ and $2 i+2$, receives two particles in the next timestep. Clearly two extra particles are created in the particle redistribution (or toppling) process. (b) for any even-numbered site $2 i$, four particles will be lost in the next timestep. Each of its two nearest neighbors, i.e. sites $2 i-1$ and $2 i+1$, receives a single particle. Thus, two particles are dissipated in the process. The odd and even-numbered sites are called creative and dissipative respectively. Open boundary conditions (i.e. particles are allowed to flow out of the system from both ends) are used. Therefore, the toppling matrix [2] of the system is given by 


$$
\Delta \equiv \Delta^{(2 N)}=\left[\begin{array}{rrrrrrr}
2 & -2 & & & & & \\
-1 & 4 & -1 & & & & \\
& -2 & 2 & -2 & & & \\
& & -1 & 4 & -1 & & \\
& & \ldots & \ldots & \ldots & \\
& & & & \ldots & \ldots & \ldots
\end{array}\right]
$$

where the superscript $(2 N)$ denotes the total number of sites in the system and hence the size of the matrix. Clearly we have constructed a one-dimensional superlattice model with equal number of dissipative and creative sites.

First we find the total number of recurrence states on the system which equals det $\Delta$ [2]. By some elementary row and column transformations, it is easy to show that

$$
\operatorname{det} \Delta^{(2 k)}=4 \operatorname{det} \Delta^{(2 k-2)}-4 \operatorname{det} \Delta^{(2 k-4)}
$$

for $k \geq 3$. Since $\operatorname{det} \Delta^{(2)}=6$ and $\operatorname{det} \Delta^{(4)}=20$, we conclude that

$$
\operatorname{det} \Delta^{(2 N)}=(2 N+1) 2^{N} .
$$

Now we want to find all the $(2 N+1) 2^{N}$ recurrence states of the system using an idea inspired by the burning algorithm [7]. We represent system configurations by row vectors of length $2 N$. It has been shown that a system configuration $\alpha \equiv\left(\alpha_{1}, \ldots, \alpha_{2 N}\right)$ is in the set of all recurrence states $\Omega$ if and only if we can find an unstable state $\beta=\left(\beta_{1}, \ldots, \beta_{2 N}\right)$ which topples to $\alpha$ with all the sites topple at least once in the process [8,9]. Clearly, $\beta_{i}=\alpha_{i}+\sum_{j} n_{j} \Delta_{j i}$ for some $n_{j} \in \mathbb{Z}^{+}$. To test if a particular state $\alpha$ is recurrence, we choose a set of $n_{i} \in \mathbb{Z}^{+}$with $a_{i}=\sum_{j} n_{j} \Delta_{j i} \geq 0$ for all $i$. The existence of such set of $n_{i}$ has been proved in Proposition 1 of Reference [10]. Nevertheless the choice of $n_{i}$ is not unique. We add $a_{i}$ particles to site $i$ for all $i$ at the same time when the system is in configuration $\alpha$. Then the resultant configuration after toppling equals $\alpha$ if and only if $\alpha$ is a recurrence state [9,11].

In the present case, we choose $n_{i}=1$ for all $i$, which is equivalent to adding a single particle to sites 1 and two particles to site $2 N$ at the same time. Since at most $\Delta_{i i}$ particles are removed from site $i$ whenever it is unstable each time, the possible local heights an odd (even) site can be when the system reaches its recurrence phase space are -1 and $0(-3,-2,-1$ and 0$)$. An odd (even) site is called "absorbing" if and only if its local height equals -1 ( -2 or -3$)$. Obviously, these are sites which can "absorb" the particles coming from their neighbors during toppling for exactly one time.

Claim: any system configuration with more than one absorbing site is not a recurrence configuration. Proof: Suppose $\alpha$ has more than one absorbing site, and the left most and right most absorbing sites are denoted by $l$ and $r$ respectively (i.e., site $i$ is not absorbing if $i<l$ or $i>r$ ). Upon addition of a particle to site 1 and two particles to site $2 N$, it is easy to verify that exactly one toppling will occur in sites $1,2, \ldots, l-2, l-1, r+1, r+2, \ldots, 2 N$. After that, the avalanche stops because both $l$ and $r$ "absorb" the incoming particles and prevent further toppling. Since $l<r$ (or else $\alpha$ has only one absorbing site), the system does not return to $\alpha$ after the avalanche. Thus $\alpha \notin \Omega$.

The remaining possible recurrence state configurations are those $\alpha$ with at most one absorbing site. The total number of such states, $T$, is given by: 


$$
T=2^{N}+\sum_{i=1}^{N} 2^{N}+\sum_{i=1}^{N} 22^{N-1}=(2 N+1) 2^{N}
$$

where the first term is the total number of stable configurations without any absorbing site, the second (the third) terms are the numbers of possible recurrence configurations with exactly one odd and no even (one even and no odd) absorbing site. Since $T=\operatorname{det} \Delta$, we conclude that stable configurations with at most one absorbing site are the only elements in the recurrence phase space $\Omega$. The above method of finding recurrence configurations is effective whenever the spatial dimension of the system is low.

Having explicitly find out all the elements in $\Omega$, we can proceed to show that the system is indeed self-organized critical. We define the avalanche size $s$ to be the total number of toppling occur during an avalanche (i.e., sites with multiple toppling are counted multiple number of times). Direct calculation tells us that if we add a single particle to site $i$ on a recurrence system configuration without any absorbing site,

$$
s=\left\{\begin{array}{cl}
i(2 N+1-i) & \text { if } h_{i}=0 \\
0 & \text { otherwise }
\end{array} .\right.
$$

Similarly, if the particle is added to a recurrence configuration with an absorbing site at $k$,

$$
s=\left\{\begin{array}{cl}
0 & \text { if } i=k \text { or } h_{i}<0 \\
(2 N+1-i)(i-k) & \text { if } i>k \text { and } h_{i}=0 \\
i(k-i) & \text { if } i<k \text { and } h_{i}=0
\end{array} .\right.
$$

So under a uniform particle addition and in the $N \rightarrow \infty$ limit, the distribution of avalanche size can be well approximated by

$$
D(s)=\left\{\begin{array}{cl}
\frac{1}{4} & \text { if } s=0 \\
\frac{3}{4} \frac{2}{N^{2}}\left[1-\frac{s}{N^{2}}\right] & \text { if } 0<s<N^{2} . \\
0 & \text { otherwise }
\end{array}\right.
$$

Thus if we Fourier transform $D(s), 1 / f^{2}$ scaling in avalanche size $s$ is observed. Therefore this model is self-organized critical although its scaling exponent is trivial.

To some extend, the above example does not completely demonstrate that particle conversation law is not a necessary condition for the exhibition of self-organized criticality in cellular automaton models. If we rescale all the even-number sites by $h_{2 i} \longrightarrow h_{2 i} / 2$, then the toppling matrix becomes that of the one-dimensional Abelian Sandpile Model. In fact, this example is equivalent to the onedimensional ASM which allows half-integral local heights for all the even-numbered sites. Besides, the even-numbered sites will receive only one half of a unit of particle each time when something is dropped onto them. The distribution of avalanche size $D(x)$ can be calculated using the piecewise linear relationship found by Chau and Ho [12] and it turns out to be the same as those given by Eqs. (5) and (6). 


\section{EXAMPLE 2}

We now provide another example on one-dimensional superlattice which is not equivalent to any "ordinary" one-dimensional sandpile models, whose toppling rules are translational invariant at all sites except possibly at system boundaries, under local rescaling. More precisely, this model is not equivalent to any one-dimensional ASM whose toppling matrix is of Toeplitz form. Again, we apply the rules of Abelian Sandpile to a collection of $2 N$ sites labeled from 1 to $2 N$. The toppling rules are given by: (a) for any odd-numbered site $2 i+1$, it will lose four particles in the next timestep. Two of them are delivered to site $2 i+3$, and one of them to site $2 i+4$ while the remaining one is dissipated in the process. Thus, odd-numbered sites are dissipative. (b) for any even-numbered site $2 i$, it will lose two particles in the next timestep. Two of them are transported to site $2 i-3$, one of them to site $2 i-2$. Thus, even-numbered sites are creative. The toppling matrix of the system is given by

$$
\Delta \equiv \Delta^{(2 N)}=\left[\begin{array}{rrrrrrrr}
4 & 0 & -2 & -1 & & & & \\
0 & 2 & & & & & & \\
& & 4 & 0 & -2 & -1 & \\
-2 & -1 & 0 & 2 & & & & \\
& & & & \ldots & \ldots & \ldots & \ldots \\
& & & \ldots & \ldots & \ldots & & \\
& & & & & & &
\end{array}\right]
$$

Using similar idea as in Example 1, it is easy to show that

$$
\operatorname{det} \Delta^{(2 N)}=(N+1) 4^{N}
$$

We use the same technique before to find all the $(N+1) 4^{N}$ recurrence states of the system. In this case, we choose $n_{i}=1$ for all $i$, which is equivalent to adding four particles to sites 1 and $2 N-1$, together with two particles to sites 2 and $2 N$ all at the same time. Since at most $\Delta_{i i}$ particles are removed from site $i$ whenever it becomes unstable each time, the possible states an even (odd) site can be when the system reaches the recurrence phase space are -1 and $0(-3,-2,-1$ and 0$)$. For any stable system configuration $\alpha \equiv\left(h_{i}\right)$, we define

$$
u_{\text {min }}=\left\{\begin{array}{cl}
i & \text { if } h_{1}, h_{3}, \ldots, h_{2 i-3} \geq-1 \text { and } h_{2 i-1}<-1 \\
N+1 & \text { if } h_{1}, h_{3}, \ldots, h_{2 N-1} \geq-1
\end{array}\right.
$$

and

$$
l_{\text {max }}=\left\{\begin{array}{l}
i \text { if } h_{2 i+2}, h_{2 i+4}, \ldots, h_{2 N}=0 \text { and } h_{2 i}=-1 \\
0 \text { if } h_{2}, h_{4}, \ldots, h_{2 N}=0
\end{array} .\right.
$$

For the odd-numbered sites, exactly two particles are received per toppling in the particles flows into them; while for the even-numbered sites, the only one particle is received per toppling. So odd-numbered sites with local height equals -3 or -2 (or even-numbered sites with local height equals -1) can "absorb" the particles coming from their neighbors during toppling for exactly once; while odd-numbered sites with local height equals -1 or 0 (or even-numbered sites with local height equals 0 ) becomes unstable whenever particles flows into them during an avalanche. Thus $2 u_{\min }-1$ 
is the minimum odd-numbered site (while $2 l_{\max }$ is the maximum even-numbered site) in our finite superlattice which can "absorb the stress" whenever particles topple onto it for exactly one time during an avalanche.

Claim: any system configuration with $u_{\min }<l_{\max }$ is not a recurrence state.

Proof: Consider a system configuration $\alpha$ with $u_{\min }<l_{\max }$. Upon addition of 2 particles to sites 1 and $2 N-1$ and 1 particle to sites 2 and $2 N$, it is easy to verify that exactly one toppling will occur in the following sites $1,2, \ldots, 2 u_{\min }-2,2 u_{\min }, 2 l_{\max }-1,2 l_{\max }+1,2 l_{\max }+2, \ldots, 2 N$. After that, the avalanche stops because both sites $2 u_{\min }-1$ and $2 l_{\max }$ "absorb" the incoming particles and prevent further toppling. Since $u_{\min }<l_{\max }$, sites such as $2 u_{\min }-1$ and $2 l_{\max }$ will not topple during the avalanche and hence after the system will not relax back to $\alpha$. Thus $\alpha \notin \Omega$.

Thus the remaining possible recurrence state configurations are those $\alpha \equiv\left(h_{i}\right)$ with $-3 \leq$ $h_{2 i-1} \leq 0$ and $-1 \leq h_{2 i} \leq 0$ for $i=1,2, \ldots, N$, and with $u_{\min } \geq l_{\max }$. The total number of such states, $T$, is given by:

$$
T=\sum_{i=1}^{N} \sum_{j=1}^{i} 2^{i-1} 24^{N-i} 2^{j-1}+\sum_{i=1}^{N} 2^{i-1} 24^{N-i}+2^{N} 2^{N}
$$

where the first term is the number of configurations with $u_{\min } \leq N$ and $l_{\max } \geq 1$, second term is the number of configurations with $l_{\max }=0$, and third term is the number of configurations with $u_{\min }=N+1$. After some computation, we find $T=(N+1) 4^{N}=\operatorname{det} \Delta$, and hence the set of all recurrence states of the system is

$$
\begin{aligned}
& \Omega=\left\{\alpha=\left(\alpha_{i}\right): \alpha_{2 j-1} \in\{-3,-2,-1,0\}, \alpha_{2 j} \in\{-1,0\}\right. \\
&\text { for } \left.i=1,2, \ldots, N \text { and } u_{\min }(\alpha) \geq l_{\min }(\alpha)\right\}
\end{aligned}
$$

Now, we go on to show that the system is indeed self-organized critical. Unlike Example 1, it is not easy to argue the distribution of avalanche size $D(s)$ owing to the complexity of the recurrence phase space $\Omega$. So we take the alternative approach by calculating the two point correlation function $G_{i j}$ of the system which is defined as the average number of toppling occurs in site $j$ given that a particle is introduced to site $i$, and is given by $G_{i j}=\Delta_{i j}^{-1}$ [2].

In the Appendix, we show that

$$
\begin{gathered}
G_{2 i, 2 j}=\left\{\begin{array}{cc}
\frac{j(N-i)}{4(N+1)} & \text { if } i>j \\
\frac{(i+1)(N+1-j)}{4(N+1)} & \text { otherwise }
\end{array}\right. \\
G_{2 i, 2 j-1}=\left\{\begin{array}{cc}
\frac{j(N-i)}{4(N+1)} & \text { if } i+1>j \\
\frac{(i+1)(N+1-j)}{4(N+1)} & \text { otherwise },
\end{array}\right. \\
G_{2 i-1,2 j}=\left\{\begin{array}{cc}
\frac{(i-1)(N+1-j)}{2(N+1)} & \text { if } i-1<j \\
\frac{j(N+2-i)}{2(N+1)} & \text { otherwise },
\end{array}\right.
\end{gathered}
$$


and

$$
G_{2 i-1,2 j-1}=\left\{\begin{array}{cl}
\frac{j(N+2-i)}{2(N+1)} & \text { if } i \geq j \\
\frac{(i-1)(N+1-j)}{2(N+1)} & \text { otherwise }
\end{array},\right.
$$

for $i, j=1,2, \ldots, N$. Obviously, the two point correlation function $G_{i j}$ varies linearly with the distance between sites $i$ and $j$. Upon a uniform and random particle addition, $1 / f^{2}$ scaling is observed as $N \rightarrow \infty$ and the model is indeed self-organized critical (but with a trivial exponent).

This model is not equivalent to any "ordinary" one-dimensional ASM with toppling rules being translational invariant except possibly at the boundary. Suppose the contrary, we can find a set of $h_{i}^{\text {new }}=f_{i}\left(h_{1}, \ldots, h_{2 N}\right)$ such that the system becomes a one-dimensional ASM after applying these transformations. $f_{i}$, however, is independent of $h_{j}$ for all $j \neq i$ or else toppling of site $i$ must depend on the neighboring sites, making the transformed system not an ASM. It is easy to check that any transformation $f_{i}=f_{i}\left(h_{i}\right)$ cannot reduce Eq. (7) to a Toeplitz form and hence this model is not equivalent to any "ordinary" one-dimensional ASM.

\section{CONCLUSIONS}

In summary, we have explicitly constructed the simplest possible class of cellular automaton examples exhibiting self-organized criticality without the presence of local particle conservation law: namely, Abelian Sandpile Models on one-dimensional superlattices. Moreover, a simple method of finding recurrence phase space configurations, based on the idea of the burning algorithm is introduced, which is useful when the dimension of the system is low.

\section{APPENDIX: APPENDIX: FINDING $\Delta^{-1}$}

We rearrange the site labels of the system using the map:

$$
\left\{\begin{array}{ccc}
2 i & \longrightarrow & i \\
2 i-1 & \longrightarrow & N+i
\end{array}\right.
$$

for $i=1,2, \ldots, N$, the toppling matrix $\Delta$ can be re-written as

$$
\Delta_{\text {new }} \equiv \Delta_{\text {new }}^{(2 N)}=\left[\begin{array}{ll}
2 D^{T} & B \\
2 B^{T} & D
\end{array}\right]
$$

where $B^{T}$ denotes the transpose of $B$. Moreover, $B$ and $D$ are $N \times N$-matrices whose elements are given by

$$
B_{i j}=\left\{\begin{aligned}
-1 & \text { if } j-i=1 \\
0 & \text { otherwise }
\end{aligned}\right.
$$

and

$$
D_{i j}=\left\{\begin{aligned}
2 & \text { if } i=j \\
-1 & \text { if } i-j=1 \\
0 & \text { otherwise }
\end{aligned}\right.
$$


respectively. According to the block matrix inversion formula,

$$
\Delta_{\text {new }}^{-1}=\left[\begin{array}{cc}
0.5 X^{-1} & -0.5\left(D^{T}\right)^{-1} B Y^{-1} \\
-D^{-1} B^{T} X^{-1} & Y^{-1}
\end{array}\right]
$$

where $X=D^{T}-B D^{-1} B^{T}$ and $Y=D-B^{T}\left(D^{T}\right)^{-1} B$. Since $D$ is a Toeplitz matrix, $D^{-1}$ can be evaluated easily [13] and is given by

$$
D_{i j}^{-1}=\left\{\begin{array}{ll}
0.5^{i-j+1} & \text { if } i \geq j \\
0 & \text { otherwise }
\end{array} .\right.
$$

As a result,

$$
X_{i j}= \begin{cases}2 & \text { if } i=j=N \\ -1 & \text { if } i-j=1 \\ 1.5 & \text { if } i=j \text { and } i<N \\ -0.5^{i-j+1} & \text { if } i>j \text { and } i<N \\ 0 & \text { otherwise }\end{cases}
$$

We divide $X$ into four block matrices by partitioning the sites into two sets, namely: $\{1,2, \ldots, N-1\}$ and $\{N\}$. The only $(N-1) \times(N-1)$-matrix so formed is in Toeplitz form whose inverse can be found readily [13]. After that, by means of the block matrix inversion formula again, we obtain

$$
X_{i j}^{-1}=\left\{\begin{array}{cl}
\frac{j(N-i)}{2(N+1)} & \text { if } i>j \\
\frac{(i+1)(N+1-j)}{2(N+1)} & \text { otherwise }
\end{array} .\right.
$$

Using the same method, we find that

$$
Y_{i j}^{-1}=\left\{\begin{array}{cl}
\frac{j(N+2-i)}{2(N+1)} & \text { if } i \geq j \\
\frac{(i-1)(N+1-j)}{2(N+1)} & \text { otherwise }
\end{array} .\right.
$$

Now $D^{-1} B^{T} X^{-1}$ and $\left(D^{T}\right)^{-1} B Y^{-1}$ can be evaluated using Eqs. (A5), (A7) and (A8). Thus all four block matrices in Eq. (A4) are computed. Finally, Eqs. (12a)-(12d) are obtained by changing the labels of the sites back to the original ones.

\section{APPENDIX: ACKNOWLEDGMENTS}

I would like to thank the Aspen Center for Physics for its hospitality during which part of the work was done. This work is supported by DOE grant DE-FG02-90ER40542. 


\section{REFERENCES}

[1] P. Bak, C. Tang, \& K. Wesienfeld, Phys. Rev. Lett. , 59, 381 (1987); P. Bak, C. Tang, \& K. Wesienfeld, Phys. Rev. A , 38, 364 (1988).

[2] D. Dhar, Phys. Rev. Lett. , 64, 1613 (1990).

[3] T. Hwa, \& M. Kardar, Phys. Rev. Lett. , 62, 1813 (1989).

[4] G. Grinstein, D. H. Lee, \& S. Sachdev, Phys. Rev. Lett. , 64, 1927 (1990); S. S. Manna, L. B. Kiss, \& J. Kertész, J. Stat. Phys. , 61, 923 (1990); K. Christensen, Z. Olami, \& P. Bak, Phys. Rev. Lett. , 68, 2417 (1992); J. E. Socolar, G. Grinstein, \& C. Jayaprakash, Phys. Rev. E , 47, 2366 (1993).

[5] K. Chen, P. Bak, \& M. H. Jensen, Phys. Lett. A , 149, 207 (1990); B. Drossel, \& F. Schwabl, Phys. Rev. Lett. , 69, 1629 (1992); S. Clar, B. Drossel, \& F. Schwabl, Phys. Rev. E , 50, 1009 (1994).

[6] P. Ruelle, \& S. Sen, J. Phys. A , 25, L1257 (1992); A. A. Ali, \& D. Dhar, cond-mat preprint \#9412085 (1994).

[7] D. Dhar, \& S. S. Manna, Phys. Rev. E , 49, 2684 (1994).

[8] E. R. Speer, J. Stat. Phys. , 71, 61 (1993).

[9] H. F. Chau, \& K. S. Cheng, J. Math. Phys. , 34, 5109 (1993).

[10] S. W. Chan, \& H. F. Chau, submitted (adap-org preprint \#9410002, 1994).

[11] H. F. Chau, Phys. Rev. E , 50, 4226 (1994).

[12] H. F. Chau, \& C. Ho, Phys. Rev. E , 49, 902 (1994).

[13] G. Heinig, \& K. Rost, Algebraic Methods for Toeplitz-like Matrices and Operators, Chap. 1 (Birkhäuser Verlag, Stuttgart, 1984). 